\title{
Does dietary intake affect age at natural menopause in the UK Women's Cohort Study?
}

\author{
Y. Dunneram ${ }^{1}$, J.E. Cade ${ }^{1}$, D.C. Greenwood ${ }^{2}$ and V.J. Burley ${ }^{1}$ \\ ${ }^{1}$ Nutritional Epidemiology Group, School of Food Science \& Nutrition and \\ ${ }^{2}$ Division of Biostatistics, University of Leeds, Leeds LS2 9JT, UK
}

Age at natural menopause is an important determinant of future health outcomes among postmenopausal women. Previous studies have demonstrated an association between a later menopause and reduced risk of cardiovascular diseases and osteoporosis, and a higher risk for endometrial, ovarian and breast cancer. ${ }^{(1)}$ It is hypothesised that diet can influence age at natural menopause. ${ }^{(2)}$ However, only a few studies have reported on the association between dietary intake and age at natural menopause.

Study participants were women aged 40-65 years who had experienced a natural menopause from the UK Women's Cohort Study (UKWCS) between baseline and first follow up. Natural menopause was defined as the permanent cessation of menstrual periods for at least 12 consecutive months. ${ }^{(3)}$ Individual foods measured using a food frequency questionnaire at baseline were collated into 64 food groups (g/day) according to culinary use, fat and fibre content. Reproductive history of participants was also recorded. Regression modelling, adjusting for confounders, was used to assess associations between diet and age at menopause. During the 4 year follow-up period, 914 women experienced a natural menopause. A high intake of oily fish and fresh legumes were associated with delayed onset of natural menopause by 3.3 years/portion ( $99 \%$ confidence interval (CI): $0 \cdot 8$ to 5.8$)$ and 0.9 years per portion ( $99 \%$ CI: 0.0 to 1.8$)$ respectively. Refined pasta and rice was associated with earlier menopause. In addition, a higher intake of vitamin B6 (per mg/day: 0.6 years, $99 \% \mathrm{CI}: 0.1$ to 1.2 ) and zinc (per mg/day: 0.3 years, $99 \% \mathrm{CI}$ : -0.0 to 0.6 ) were associated with later age at menopause. Women who were vegetarian had earlier age at natural menopause by 0.8 years compared to non-vegetarians (99\% CI: $0 \cdot 2$ to 1.4$)$.

\begin{tabular}{|c|c|c|c|c|c|c|}
\hline Daily intake & $\begin{array}{c}\text { Difference in age at natural } \\
\text { menopause }(\mathrm{y})^{\dagger}\end{array}$ & $99 \% \mathrm{CI}$ & $P$ & $\begin{array}{l}\text { Difference in age at natural } \\
\text { menopause }(y)^{*}\end{array}$ & $99 \% \mathrm{CI}$ & $P$ \\
\hline \multicolumn{7}{|l|}{ Food groups (portion size) } \\
\hline Refined pasta and rice $(210 \mathrm{~g})$ & $-1 \cdot 8$ & $-3 \cdot 0$ to -0.5 & $<0 \cdot 001$ & $-1 \cdot 5$ & $-2 \cdot 8$ to -0.2 & 0.003 \\
\hline Oily fish $(90 \mathrm{~g})$ & $3 \cdot 2$ & 0.8 to $5 \cdot 6$ & 0.001 & $3 \cdot 3$ & 0.8 to 5.8 & 0.001 \\
\hline Fresh legumes (75 g) & 1.0 & 0.1 to 1.8 & $0 \cdot 003$ & $0 \cdot 9$ & 0.0 to 1.8 & 0.007 \\
\hline \multicolumn{7}{|l|}{ Nutrients (mg) } \\
\hline Vitamin $\mathrm{B}_{6}$ & $0 \cdot 4$ & -0.0 to 0.7 & $0 \cdot 014$ & $0 \cdot 6$ & 0.1 to 1.2 & 0.005 \\
\hline Zinc & $0 \cdot 2$ & $-0 \cdot 0$ to $0 \cdot 3$ & $0 \cdot 012$ & $0 \cdot 3$ & -0.0 to 0.6 & 0.007 \\
\hline
\end{tabular}

Unadjusted model; *Adjusted for smoking status, alcohol consumption, physical activity, social class.

Our findings have demonstrated for the first time that some food groups as well as specific nutrients are individually predictive of age at natural menopause in a cohort of British women. These findings will contribute to an improved understanding of the timing of natural menopause in relation to diet, and may also have implications associated with longer term health outcomes in postmenopausal women.

This work was supported by the Commonwealth Scholarships, UK.

1. Gold EB (2011) Obstet Gynecol Clin North Am 38, 425-440.

2. Nagata C, Takatsuka N, Inaba S et al. (1998) Maturitas 29, 105-113.

3. Schoenaker DA, Jackson CA, Rowlands JV et al. (2014) Int J Epidemiol 43, 1542-1562. 